\title{
Theoretical insights into the cycloaddition reaction mechanism between ketenimine and methyleneimine: An alternative approach to the formation of pyrazole and imidazole
}

\author{
NANA WANG ${ }^{\mathrm{a}}$, XIAOJUN TAN $^{\mathrm{b}, *}$, WEIHUA WANG ${ }^{\mathrm{c}}$, FANGFANG WANG $^{\mathrm{b}}$ and PING LI ${ }^{\mathrm{c}, *}$ \\ a'Yantai Automobile Engineering Professional College, Yantai, Shandong, 265500, People's Republic of China \\ ${ }^{b}$ College of Biological Science and Technology, University of Jinan, Jinan, Shandong, 250022, \\ People's Republic of China \\ ${ }^{\mathrm{c}}$ School of Chemistry and Chemical Engineering, Qufu Normal University, Qufu, Shandong, 273165, \\ People's Republic of China \\ e-mail: chem.2001@163.com; lpsdu@sdu.edu.cn
}

MS received 25 August 2015; revised 1 November 2015; accepted 15 December 2015

\begin{abstract}
The cycloaddition reaction mechanism between interstellar molecules, ketenimine and methyleneimine, has been systematically investigated employing the second-order Møller-Plesset perturbation theory (MP2) method in order to better understand the reactivity of nitrogenous cumulene ketenimine with the $\mathrm{C}=\mathrm{N}$ double bond compound methyleneimine. Geometry optimizations and vibrational analyses have been performed for the stationary points on the potential energy surfaces of the system. Calculations show that five-membered cyclic carbene intermediates could be produced through pericyclic reaction processes between ketenimine and methyleneimine. Through the subsequent hydrogen transfer processes, carbene intermediates can be isomerized to the pyrazole and imidazole compounds, respectively. The present study is helpful to understand the formation of prebiotic species in interstellar space.
\end{abstract}

Keywords. Ketenimine; methyleneimine; reaction mechanism; interstellar molecule.

\section{Introduction}

Ketenimine, $\mathrm{CH}_{2}=\mathrm{C}=\mathrm{NH}$, has attracted much attention as a reactive intermediate in organic chemistry ${ }^{1}$ and astrochemistry. ${ }^{2}$ Jacox et al., predicted in 1963 that ketenimine is tentatively identified as a product of the reaction of the imidyl radical $(\mathrm{NH})$ with acetylene in solid argon. ${ }^{3}$ In 1979, Jacox accomplished the first spectroscopic identification of ketenimine with the matrix isolation study of the products from the reaction of excited argon atom with acetonitrile $\left(\mathrm{CH}_{3} \mathrm{CN}\right){ }^{4}$ The first study in gas phase, by microwave spectroscopy, was reported by Rodler et al., in 1984, in which 2cyanoethanol $\left(\mathrm{HOCH}_{2} \mathrm{CH}_{2} \mathrm{CN}\right)$ was pyrolized at $800^{\circ} \mathrm{C}$ to form ketenimine. ${ }^{5}$

Ketenimine has been detected in the star-forming region Sagittarius B2(N) by means of radio telescopes. It is a relatively abundant species in Sgr B2(N) and is likely formed directly from its isomer acetonitrile by tautomerization driven by shocks that pervade the

\footnotetext{
${ }^{*}$ For correspondence
}

star-forming region. ${ }^{2}$ Therefore, several theoretical and experimental investigations have been performed to study the formation of ketenimine in the interstellar media. Nadia reported the combined crossed beam and theoretical studies on the $\mathrm{N}\left({ }^{2} \mathrm{D}\right)+\mathrm{C}_{2} \mathrm{H}_{4}$ reaction and implications for atmospheric models of Titan. ${ }^{6}$ They verified that the ketenimine is one of the main products for this reaction. Given that nitrile derivatives have been detected in Titan's atmosphere, cometary comas, and the interstellar media, Hudson et al., reported experimental investigations on the low-temperature chemistry of some of these compounds. In the absence of water, ketenimine is one of the photo- and radiochemical products of these nitriles. ${ }^{7}$ Aminoacetonitrile, a species of astrochemical interest, has been detected in the interstellar media. The reaction of aminoacrylonitrile with $\mathrm{Ni}^{+}$was investigated by means of mass spectrometry techniques and density functional theory calculations, where the ketenimine is one of the main products for this reaction. ${ }^{8}$ Using Fourier transform infrared spectroscopy, ketenimine is trapped and identified by UV-irradiation of $\mathrm{CH}_{3} \mathrm{COCN}$ in an argon matrix at $10 \mathrm{~K} .{ }^{9}$ 
As an unstable nitrogenous cumulene, ketenimine can react with unsaturated compounds to form heterocyclic compounds. Liu et al., investigated the cycloaddition reaction between ketenimine and cyclopentadiene theoretically, establishing that the activation energy for this reaction is $156.5 \mathrm{~kJ} / \mathrm{mol}$ using the G3B3 method. ${ }^{10}$ Fang et al., studied the formation of four-membered compounds through the stepwise cycloaddition reaction between ketenimine and olefins using $a b$ initio methods. ${ }^{11}$ In addition, Sun et al., reported the reaction between ketenimine and water, where acetamide is finally formed by tautomerization. ${ }^{12}$ Sung et al., reported the amination reaction of ketenimine employing NMR and $a b$ initio studies, which provided the formation pathway of vinylidenediamine. ${ }^{13} \mathrm{Wu}$ et al., studied the stepwise mechanism between ketenimine and methyleneimine in the gas phase theoretically. ${ }^{14}$ It was found that three four-membered cyclic products, 2-azetidinimine, 2methylene-1,3-diazetidine, and 3-azetidinimine can be formed and the energy barriers of controlling steps for the three pathways are $137.5,122.8$, and $310.6 \mathrm{~kJ} / \mathrm{mol}$ at the HF/6-31G** level of theory $(69.4,105.3$, and $162.8 \mathrm{~kJ} / \mathrm{mol}$ at the MP2/6-31G** level of theory), respectively. However, the stabilities of those compounds have not been evaluated. ${ }^{14}$

The fact that organic chemistry started in space can be considered as a new challenge for the scientific community. Among the extrasolar objects, the Galactic center molecular clouds, ${ }^{15}$ hot cores, ${ }^{16}$ and hot corinos ${ }^{17}$ are particularly rich in complex organic molecules (COMs). Some more complicated COMs have been observed in comets ${ }^{18}$ and meteorites, where amino acids have also been detected. ${ }^{19}$ Ketenimine and methyleneimine are both interstellar molecules. ${ }^{20}$ Accordingly, we postulate that the reaction of ketenimine and methyleneimine may be one of the formation pathways of prebiotic species. In the present study, we have performed comprehensive theoretical investigations on the reaction mechanism between ketenimine and methyleneimine employing the second-order Møller-Plesset perturbation theory (MP2) method in order to better understand the ketenimine reactivity and possible formation of prebiotic species in the interstellar space.

\section{Computational method}

The second-order Møller-Plesset perturbation theory (MP2) method in combination with the $6-311+\mathrm{G}^{*}$ basis set has been employed to locate all the stationary points along the reaction pathways without imposing any symmetry constraints. Frequency analyses have been carried out to confirm the nature of the minima and transition states. Moreover, intrinsic reaction coordinate (IRC) calculations have also been performed to further validate the calculated transition states connecting reactants and products. Additionally, the relevant energy quantities, such as the reaction energies and barrier heights, have been corrected with the zero-point vibrational energy (ZPVE) corrections.

To further refine the calculated energy parameters, single point energy calculations for all pathways have been performed at the $\operatorname{CCSD}(\mathrm{T}) / 6-311+\mathrm{G}^{*}$ level of theory based on the optimized geometries at the MP2/6$311+\mathrm{G}^{*}$ level of theory. To evaluate the influence of basis set on the calculated energy parameters, we have also carried out the relevant computations employing the correlated basis set, aug-cc-pVTZ. As summarized in table 1, all these levels can give consistent results for the calculated reaction profile. For the sake of simplicity, the energetic results at the $\operatorname{CCSD}(\mathrm{T}) / / \mathrm{MP} 2 / 6-$ $311+\mathrm{G}^{*}$ level of theory have been mainly discussed below if not noted otherwise.

All the calculations have been performed using Gaussian 98 program. $^{21}$

\section{Results and Discussion}

As displayed in scheme 1, four possible pathways for the title reaction have been proposed. The geometric parameters for the reactants (R1-ketenimine and R2methyleneimine), transition states (TS), intermediates (IM), and products (P) involved in the pathways (1), (2), (3), and (4) are displayed in figure 1. The calculated relative energies for the available stationary points have been summarized in table 1 . The corresponding reaction profile is illustrated in figure 2 .

\subsection{Step (a): pericyclic reaction process to form a five-membered cyclic carbene intermediate IMa}

For the pathways (1) and (2), the first intermediate IMa is formed via a pericyclic reaction process with an energy barrier of $122.1 \mathrm{~kJ} / \mathrm{mol}$. The unique imaginary frequency calculated for the corresponding transition state, TSa, in the step (a) is $509 \mathrm{i} \mathrm{cm}^{-1}$ at the $\mathrm{MP} 2 / 6-311+\mathrm{G}^{*}$ level of theory.

As shown in figure 1 , in TSa, the distances of $\mathrm{C}^{1}$ $\mathrm{N}^{2}$ and $\mathrm{N}^{1}-\mathrm{C}^{3}$ are 1.910 and $2.186 \AA$, respectively. Thus, two new bonds, namely $\mathrm{C}^{1}-\mathrm{N}^{2}$ and $\mathrm{N}^{1}-\mathrm{C}^{3}$, are being formed in the transition state TSa. At the same time, the distance of $\mathrm{N}^{2}-\mathrm{C}^{3}$ in $\mathrm{R} 2$ fragment of TSa reached $1.332 \AA$, which is $0.051 \AA$ longer than that in 
Table 1. The calculated relative energies (in $\mathrm{kJ} / \mathrm{mol}$ ) with respect to the isolated reactants at the MP2/6-311+G* and MP2/aug-cc-pVDZ (after the slash) levels of theory considering the ZPVE corrections ${ }^{a}$.

\begin{tabular}{lcc}
\hline Pathways & \multicolumn{2}{c}{ Relative Energies } \\
\hline & TSa & IMa \\
Pathway (1) & $112.3(122.1) / 104.1$ & $-2.1(-1.1) / 0.3$ \\
TS1 & $-106.3(-91.8) /-106.2$ \\
Pathway (2) & $117.3(127.4) / 110.7$ & PS2 \\
& $158.7(170.4) / 147.8$ & $-151.3(-139.2) /-144.8$ \\
& TSb & $59.0(56.5) / 60.6$ \\
& $96.9(134.3) / 84.8$ & P3 \\
PS3 & $-43.6(-34.3) /-43.7$ \\
Pathway (3) & $209.5(215.4) / 200.6$ & TS4 \\
Pathway (4) & $202.7(221.1) / 193.8$ & $-112.9(-101.8) /-105.5$ \\
&
\end{tabular}

${ }^{a}$ The data in parentheses refer to the results at the $\operatorname{CCSD}(\mathrm{T}) / / \mathrm{MP} 2 / 6-311+\mathrm{G}^{*}$ level of theory considering the ZPVE corrections.

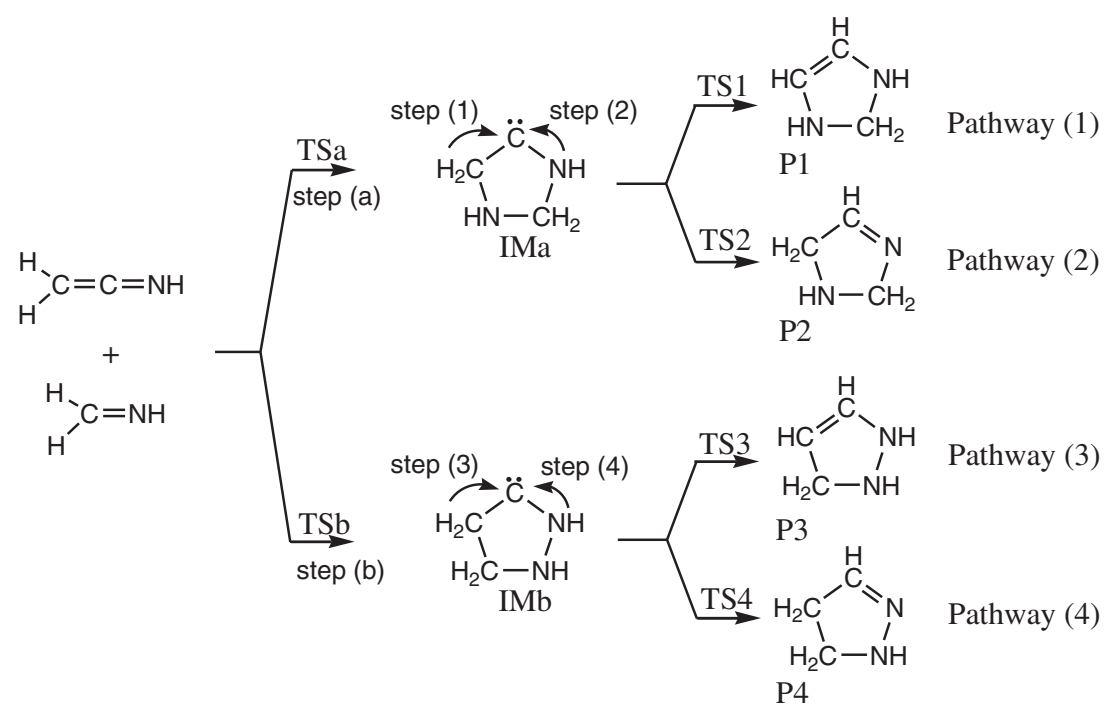

Scheme 1. The proposed four pathways for the cycloaddition reaction between ketenimine and methyleneimine.

methyleneimine. Based on the bond length data, the double bond $\mathrm{N}^{2}-\mathrm{C}^{3}$ in methyleneimine is being transformed into a single bond in TSa. Moreover, the bond angle $\mathrm{C}^{1} \mathrm{C}^{2} \mathrm{~N}^{1}$ decreased continuously. The formation of new $\sigma$ bonds of $\mathrm{C}^{1}-\mathrm{N}^{2}$ and $\mathrm{N}^{1}-\mathrm{C}^{3}$ and the cleavage of $\pi$ bond of $\mathrm{N}^{2}-\mathrm{C}^{3}$ occurred simultaneously. Therefore, the formation of IMa is a concerted reaction process. Certainly, as commented by one reviewer, the above process could also take place in an asynchronous fashion from the viewpoint of the orbital overlap due to the unsymmetry of the both reactants. As shown in figure 3, those changes of bond lengths and bond angle can be further validated by an IRC calculation on the basis of TSa.
Qualitatively, the pericyclic reaction process can be understood from the frontier molecular orbital theory. As displayed in figure 4, the frontier orbitals of HOMO of ketenimine and LUMO+2 of methyleneimine are symmetric matching, where the orbital energies have been obtained at the MP2/6-311+G* level of theory. In addition, the HOMO energy ( -0.35643 a.u.) of ketenimine is close to the LUMO+2 energy ( 0.09108 a.u.) of methyleneimine. Therefore, ketenimine can react with methyleneimine through pericyclic process to form the five-membered intermediate IMa.

In IMa, there is a lone electron pair on $\mathrm{C}^{2}$, suggesting that it is a carbene in nature. It is well known that carbene species are usually unstable. Actually, the 


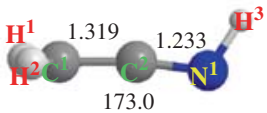

R1-ketenimine

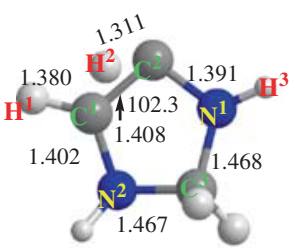

TS1

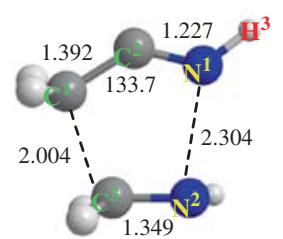

$\mathrm{TSb}$

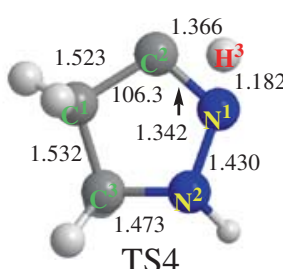

TS4

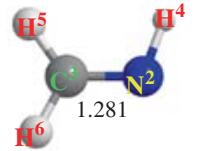

R2-methyleneimine

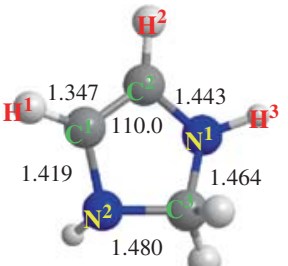

P1
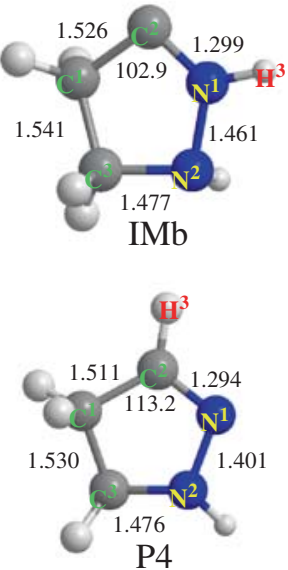

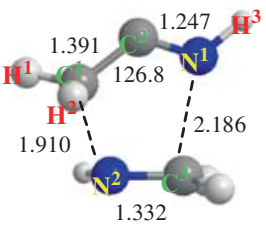

$\mathrm{TSa}$

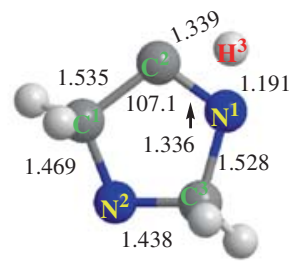

TS2

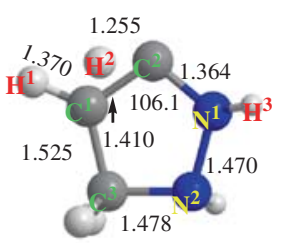

TS3

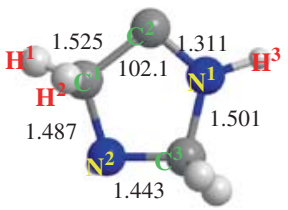

IMa

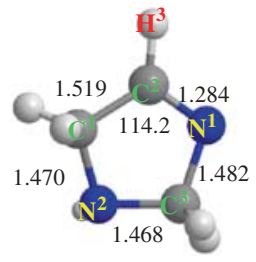

P2

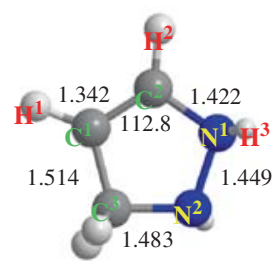

P3

Figure 1. Optimized structures of the reactants (ketenimine and methyleneimine), transition states (TS), intermediates (IM), and product (P) in the four reaction pathways at the MP2/6-311+G* level of theory, where the bond length and bond angle are in angstrom and degree, respectively.

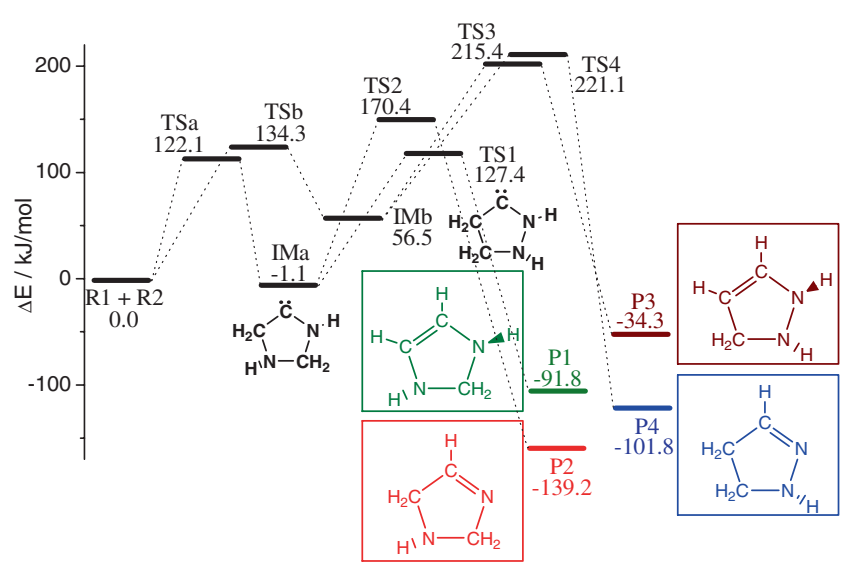

Figure 2. Reaction profile for the reaction between ketenimine and methyleneimine at the $\operatorname{CCSD}(\mathrm{T}) / / \mathrm{MP} 2 / 6-311+\mathrm{G}^{*}$ level of theory.

formation of the intermediate IMa is exothermic slightly by $1.1 \mathrm{~kJ} / \mathrm{mol}$, compared with the energy of the separated reactants. In general, IMa will be isomerized to

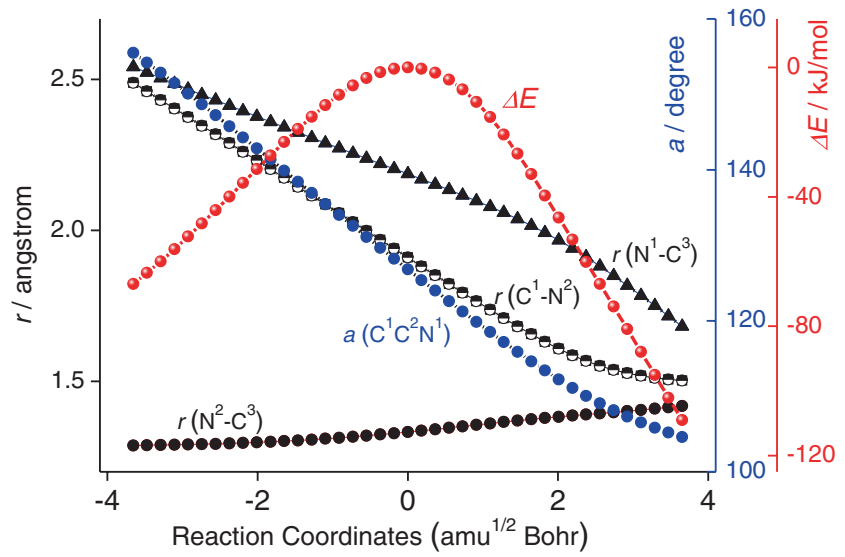

Figure 3. The selected bond lengths, bond angle, and energy changes along the reaction coordinates of step (a) between ketenimine and methyleneimine.

the stable species by bonding its lone electrons. Therefore, the next step of the reaction is the hydrogen transfer process, followed by the formation of the products 


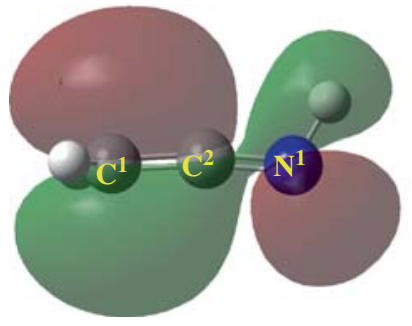

HOMO of ketenimine

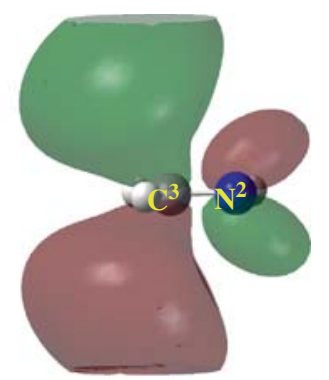

$\mathrm{LUMO}+2$ of methyleneimine
Figure 4. The calculated molecular orbitals for ketenimine and methyleneimine.

P1 and P2, respectively. It should be noted that IMa is treated as a singlet here. Corresponding triplet intermediate is also calculated as well, where it is higher in energy by $212.7 \mathrm{~kJ} / \mathrm{mol}$ than that of IMa. Therefore, the present calculations are based on the singlet intermediate IMa.

\subsection{Step (1): Hydrogen transfer process to form P1 (2,3-dihydro-1H-imidazole)}

The reaction step (1) is the transfer of the hydrogen $\mathrm{H}^{2}$ from $\mathrm{C}^{1}$ to the adjacent $\mathrm{C}^{2}$, resulting in the isomerization of IMa into P1 via TS1. Here, the calculated barrier is $128.5 \mathrm{~kJ} / \mathrm{mol}$, and the imaginary frequency of TS1 is $1254 \mathrm{i} \mathrm{cm}^{-1}$. In details, as shown in figure 1 , the distance of $\mathrm{C}^{1}-\mathrm{H}^{2}$ in TS1 has been elongated to $1.380 \AA$, and the distance of $\mathrm{C}^{2}-\mathrm{H}^{2}$ reached $1.311 \AA$, which indicates that the $\mathrm{H}^{2}$ atom is being transfered from $\mathrm{C}^{1}$ to $\mathrm{C}^{2}$. At the same time, the bond length of $\mathrm{C}^{1}-\mathrm{C}^{2}$ in TS1 decreases to $1.408 \AA$ (the bond length of $\mathrm{C}^{1}-\mathrm{C}^{2}$ in IMa is $1.525 \AA$ ), suggesting that the single bond of $\mathrm{C}^{1}-\mathrm{C}^{2}$ in IMa is becoming a double bond in P1. As shown in figure 5 , those changes of bond lengths have been validated by means of an IRC calculation on TS1.

As for P1, it is 2,3-dihydro- $1 H$-imidazole. All the cyclized atoms are in the same plane. Unlike the IMa, there are no lone electron pair in P1. Along the reaction profile, $\mathrm{P} 1$ is the relatively stable species, which is exothermic by $91.8 \mathrm{~kJ} / \mathrm{mol}$ compared with the separated reactants.

\subsection{Step (2): Hydrogen transfer process to form P2 (2,5-dihydro-1H-imidazole)}

Similar to the reaction step (1), the step (2) is also a hydrogen transfer process. In the step (2), $\mathrm{H}^{3}$ atom in IMa migrates from $\mathrm{N}^{1}$ to $\mathrm{C}^{2}$, and IMa converts to $\mathrm{P} 2$

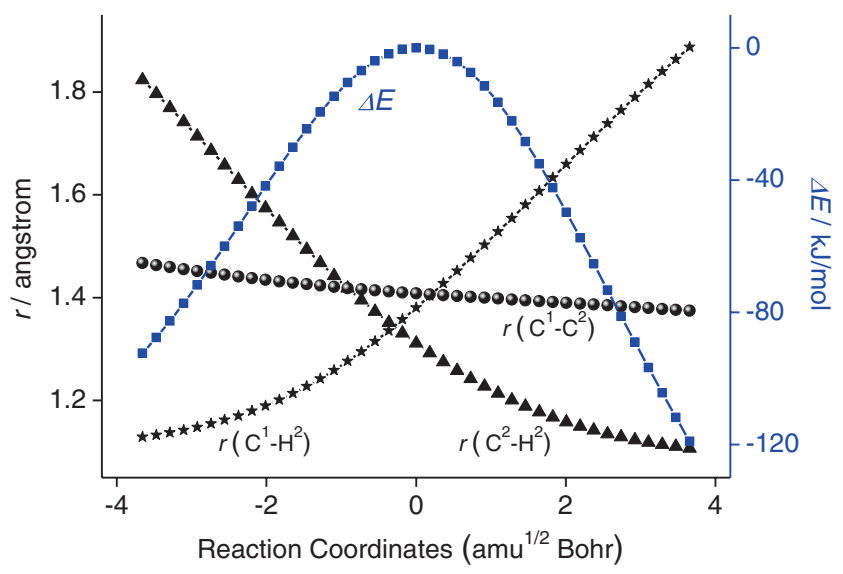

Figure 5. The selected bond lengths and energy changes along the reaction coordinates of step (1) between ketenimine and methyleneimine.

via TS2 with the reaction barrier of $171.5 \mathrm{~kJ} / \mathrm{mol}$. P2, namely 2,5-dihydro- $1 H$-imidazole, is the most stable species, which is exothermic by $139.2 \mathrm{~kJ} / \mathrm{mol}$ compared with the isolated reactants.

\subsection{Pathways (3) and (4) to form pyrazole products}

Similar to the description in section 3.1, ketenimine can react with methyleneimine to form the $\mathrm{C}^{1}-\mathrm{C}^{3}$ and $\mathrm{N}^{1}$ $\mathrm{N}^{2} \sigma$ bonds as well. The formed intermediate IMb can be isomerized to $\mathrm{P} 3$ and $\mathrm{P} 4$ through the hydrogen transfer processes, which are named as reaction pathways (3) and (4). The geometric parameters for the reactants, transition states, intermediates, and products involved in the reaction pathways (3) and (4) are displayed in figure 1 . The calculated relative energies for the available stationary points have been summarized in table 1 .

The barrier of the first step (i.e., concerted reaction process to form a singlet carbene intermediate $\mathrm{IMb}$ ) of pathways (3) and (4) is $134.3 \mathrm{~kJ} / \mathrm{mol}$, which is $12.2 \mathrm{~kJ} / \mathrm{mol}$ higher than that of the pathways (1) and (2). Here, the corresponding triplet intermediate is also calculated, where it is higher in energy by $208.1 \mathrm{~kJ} / \mathrm{mol}$ than IMb. Therefore, the following calculations are based on the singlet intermediate IMb. Because the electronegativity of nitrogen atom is strong, it is a little more difficult for two $\mathrm{N}$ atoms to approach each other to form a new $\sigma$ bond than the reaction between $\mathrm{N}$ and $\mathrm{C}$ atoms to form the $\sigma$ bond. Subsequently, the following processes of pathways (3) and (4) are similar to the pathways (1) and (2), that is, hydrogen transfer processes can lead to the formations of P3 in step (3) and P4 in step (4), which are named as 2,3-dihydro- $1 H$ pyrazole and 4,5-dihydro- $1 H$-pyrazole, respectively. 


\subsection{Comparisons of the four reaction pathways}

As mentioned above, concerted reaction steps (a) and (b) give rise to the carbene imidazole and pyrazole intermediates for which the energy barriers are 122.1 and $134.3 \mathrm{~kJ} / \mathrm{mol}$, respectively. Therefore, the carbene imidazole intermediate is easy to be formed. For the formaiotns of P1 in step (1) and P2 in step (2), the corresponding energy barriers are 128.5 and $171.5 \mathrm{~kJ} / \mathrm{mol}$, respectively. Therefore, from the kinetic viewpoint, the reaction pathway (1) is the most favorable channel. For the final products (P1 and P2) in the pathways (1) and (2), their energies are -91.8 and $-139.2 \mathrm{~kJ} / \mathrm{mol}$, respectively. Therefore, from the thermodynamic viewpoint, P2 (2,5-dihydro- $1 H$-imidazole) is the dominant product. As for the pathways (3) and (4), it is slightly difficult for ketenimine to react with methyleneimine through the pericyclic reaction step. Therefore, pathways (1) and (2) are the primary reaction processes.

Calculations and observations show that the most stable isomer is always the most abundant when several isomers of the same generic formula are identified. Moreover, the abundance ratio of the most stable isomer to the other isomers is directly related to their energy difference. This can be seen as the minimum energy principle, which has been verified in molecular clouds, hot cores/corinos, photodissociation regions, and asymptotic giant branch stars. ${ }^{22}$ Therefore, for the products of reaction between ketenimine and methyleneimine, P2 (2,5-dihydro- $1 H$-imidazole) should be more abundant than other isomers.

\section{Conclusions}

In this study, the cycloaddition reaction mechanisms between ketenimine and methyleneimine have been systematically investigated at the MP2/6-311+G* levels of theory. It was found that the five-membered cyclic carbene intermediates can be formed by means of a pericyclic reaction between ketenimine and methyleneimine. Followed by hydrogen transfer process, carbene intermediates can be isomerized to imidazole and pyrazole products, respectively. There are four pathways (1), (2), (3), and (4) to form four ultimate products P1 (2,3-dihydro- $1 H$-imidazole), P2 (2,5-dihydro- $1 H$-imidazole), P3 (2,3-dihydro- $1 H$ pyrazole), and $\mathrm{P} 4$ (4,5-dihydro- $1 H$-pyrazole), respectively. From the kinetic viewpoint, the reaction pathway (1) is the most favorable channel. From the thermodynamic viewpoint, P2 is the dominating product. Given that most organic molecules with physiological activity have a heterocyclic constituent, the heterocyclic products (imidazole and pyrazole) obtained in the reaction between ketenimine and methyleneimine in the interstellar space may play important roles in the origin of prebiotic species.

\section{Supporting Information (SI)}

Atomic coordinates and other data for the molecules are available at www.ias.ac.in/chemsci.

\section{Acknowledgements}

This work is supported by NSFC (21003082, 21303093, 21577076, 21403088), the NSF of Shandong Province (ZR2014BM020), and the project of Shandong Province Higher Educational Science and Technology Program (J13LM06). The State Key Laboratory of Environmental Chemistry and Ecotoxicology, Research Center for Eco-Environmental Sciences, Chinese Academy of Sciences (KF2013-05) are also acknowledged.

\section{References}

1. Cláudio M N, Igor R, Teresa M D, Pinhoe M, Rui F, Tomáš Š and Thomas B $2011 \mathrm{~J}$. Am. Chem. Soc. 50 18911

2. Lovas F J, Hollis J M, Remijan A J and Jewell P R 2006 Astrophys. J. 2 L137

3. Jacox M E and Milligan D E 1963 J. Am. Chem. Soc. 3 278

4. Jacox M E 1979 Chem. Phys. 2157

5. Rodler M, Brown R D, Godfrey P D and Tack L M 1984 Chem. Phys. Lett. 5447

6. Nadia B, Dimitrios S, Francesca L, Raffaele P, Mathias H, Wolf D G and Piergiorgio C 2012 J. Phys. Chem. A 4310467

7. Hudson R L and Moore M H 2004 Icarus 172466

8. Lamsabhi A M, Otilia M, Manuel Y, Salpin J Y, Haldys V, Tortajada J and Guillemin J C 2008 J. Phys. Chem. A 4210509

9. Guennoun Z, Couturier-Tamburelli I, Combes S, Aycard J P and Piétri N 2005 J. Phys. Chem. A 5111733

10. Liu S, Lei Y, Qi X T and Lan Y 2014 J. Phys. Chem. A 142638

11. Fang D C and Li H M 2000 J. Mol. Struc. (Theochem) 528111

12. Sun X M, Wei X G, Wu X P, Ren Y, Wong N B and Li W K 2010 J. Phys. Chem. A 1595

13. Sung K, Wu S H, Wu R R and Sun S Y 2002 J. Org. Chem. 124298

14. Wu F and He S R 2000 Acta Phys-Chim. Sin. 16243

15. Hollis J M, Remijan A J, Jewell P R and Lovas F J 2006 Astrophys. J. 2933

16. Ikeda M, Ohishi M, Nummelin A, Dickens J E, Bergman P, Hjalmarson $\AA$ and Irvine W M 2001 Astrophys. J. 2 792

17. Bottinelli S, Ceccarelli C, Williams J P and Lefloch B 2007 Astron. Astrophys. 2601 
18. Scott A S, Jérôme A, Conel M, Tohru A, Saša B, Giuseppe A B, Janet B, John P B, Donald E B, John R B, Mark J B, Henner B, Anna B, Simon J C, George C, Luigi C, George C, Louis D, Zahia D, Jason P D, Gianluca F, Holger F, George J F, Ian A F, Marc F., Mary K G, Daniel P G, Matthieu G, Faustine G, Chris J, Lindsay P K, Kilcoyne A L, Jan L, Matrajt G, Meibom A, Mennella V, Mostefaoui S, Nittler L R, Palumbo M E, Papanastassiou D A, Robert F, Rotundi A, Snead C J, Spencer M K, Stadermann F J, Steele A, Stephan T, Tsou P, Tyliszczak T, Westphal A J, Wirick S, Wopenka B, Yabuta H, Zare R N and Zolensky M E 2006 Science 3141720

19. Pizzarello S, Huang Y, Becker L, Poreda R J, Nieman R A, Cooper G and Williams M 2001 Science 2932236

20. Remijan A J, Hollis J M, Lovas F J, Plusquellic D F and Jewell P R 2005 Astrophys. J. 632333
21. Frisch M J, Trucks G W, Schlegel H B, Scuseria G E, Robb M A, Cheeseman J R, Zakrzewski V G, Montgomery J A, Stratmann R E, Burant J C, Dapprich S, Millam J M, Daniels A D, Kudin K N, Strain M C, Farkas O, Tomasi J, Barone V, Cossi M, Cammi R, Mennucci B, Pomelli C, Adamo C, Clifford S, Ochterski J, Petersson G A, Ayala P Y, Cui Q, Morokuma K, Malick D K, Rabuck A D, Raghavachari K, Foresman J B, Cioslowski J, Ortiz J V, Stefanov B B, Liu G, Liashenko A, Piskorz P, Komaromi I, Gomperts R, Martin R L, Fox D J, Keith T, Al-Laham M A, Peng C Y, Nanayakkara A, Gonzalez C, Challacombe M, Gill P M, Johnson B G, Chen W, Wong M W, Andres J L, HeadGordon M, Replogle E S and Pople J A 1998 Gaussian 98, Revision A.9 (Gaussian Inc., Pittsburgh, PA)

22. Lattelais M, Pauzat F, Ellinger Y and Ceccarelli C 2009 Astrophys. J. 696 L133 